\title{
On the estimation of stress strength reliability parameter of inverted exponential distribution
}

\author{
Sanjay Kumar Singh ${ }^{1}$, Umesh Singh ${ }^{1}$, Abhimanyu Singh Yadav ${ }^{2 *}$, Pradeep Kumar Vishwkarma ${ }^{2}$ \\ ${ }^{1}$ Professor, Department of Statistics and DST-CIMS, Banaras Hindu University, Varanasi-221005 \\ ${ }^{2}$ Research Scholar, Department of Statistics and DST-CIMS, Banaras Hindu University, Varanasi-221005 \\ *Corresponding author, Email: asybhu10@gmail.com
}

Copyright (C2015 Sanjay Kumar Singh et. al. This is an open access article distributed under the Creative Commons Attribution License, which permits unrestricted use, distribution, and reproduction in any medium, provided the original work is properly cited.

\begin{abstract}
This paper aims to estimate the stress-strength reliability parameter $R=P(Y<X)$, when $X$ and $Y$ are independent inverted exponential random variable. We have also disscused some fundamental properties of the considered distribution. The maximum likelihood estimator (MLE) of $R$ and its asymptotic distribution are obtained. The Bayesian estimation of the reliability parameter has been also discussed under the assumption of independent gamma prior. Numerical integration technique is used for Bayesian computation. The proposed estimators are compared in terms of their mean squared errors through the simulation study. Two real data sets representing survival of head and neck cancer patients are fitted using the inverted exponential distribution and used to estimate the stress-strength parameters and reliability.
\end{abstract}

Keywords: Inverted exponential distribution, Inverse Moment estimate and generating function, Stochastic Ordering, Renyi entropy and Bayesian inferences.

\section{Introduction}

The exponential distribution is the most exploited distribution in survival analysis, due to its simplicity and mathematical feasibility. The applicability of this distribution is restricted due to its constant failure rate pattern and seems to be less advantageous when failure rate is non-constant. Therefore, a number of lifetime models such as Weibull, gamma, generalized exponential etc. have been introduced in literature, which passes the properties of monotonicity of failure rate function. In many real life situation the pattern of non-monotonicity of the hazard rate has been observed. For example, in the course of the study of mortality associated with some of the diseases, the failure rate initially increases with time and reaches a peak after some finite period of times and then decline slowly see [9]. Thus, for analyse such type of data Inverted exponential distribution (IED) has been proposed as a lifetime model by [7]. [7], [8], [9] have advocated the use of IED as an appropriate model for non-monotonic satiation and also discussed the estimation procedure. [8] has discussed the properties of the various estimators for the IED and also presented the estimation of the parameter, based on lower record values. [9] proposed Bayes estimators of the parameter and reliability function for the same under general entropy loss function for complete, Type-I and Type-II censored samples. The applicability of inverted exponential distribution has been also seen in presence of hybrid censored data, see [16]. Recently, [17] used this model for estimation of the parameter and reliability characteristics under progressive type-II censoring scheme. The probability density function (pdf) and cumulative 
distribution function (cdf) of inverted exponential distribution (IED) are given as;

$f(x, \alpha)=\frac{\alpha}{x^{2}} e^{-\alpha / x} \quad ; x \geq 0, \alpha>0$

and

$F(x, \alpha)=e^{-\alpha / x} \quad ; x \geq 0, \alpha>0$

respectively.

The main aim of this paper is two fold. Firstly, we have discussed some fundamental properties of the considered distribution. Secondly, we develop the estimation procedure for the stress-strength parameter $R=P(Y<X)$, when, $X$ and $Y$ are independent $I E D\left(\alpha_{1}\right)$ and $I E D\left(\alpha_{2}\right)$ r. v. respectively.

The estimation of stress-strength parameter plays an important role in the reliability analysis. For example if $X$ is the strength of a system which is subjected to stress $Y$, then the parameter $R$ measures the system performance which is frequently used in the context of mechanical reliability of a system. Moreover, $R$ provides the probability of a system failure, if the system fails whenever the applied stress is greater than its strength. This problem has a long history starting with the pioneering work of [23] and [24]. The term stress-strength was first introduced by [22]. Since then significant amount of work have been done both from parametric and non-parametric point of view. This particular problem was also considered by [18] and later by [15]. In both cases it is assumed that the common location parameter is known. In the literature the estimation of $R$ in the case of Weibull or exponential distributions has been obtained under the assumption of known location parameters, see, [19]. Some of the recent work on the stress-strength model can be seen in [12], [13], [14] and [20] etc.

The paper has been organized in the following sections. Section 2 provides some mathematical properties of the IED model. Maximum likelihood estimate of the stress-strength reliability along with their asymptotic distributions are presented in Sections 3, $4 \& 5$ respectively. Bayes estimators under independent informative and non-informative gamma prior are discussed in section 6 . Section 7 , illustrate an application of the above study in real life application. Section 8, presents the simulation result to study the behaviour of the proposed estimators. Section 9, provides final concluding remarks of the considered study.

\section{Basic properties of IED}

\subsection{Moment}

The $r$ - th moment about origin is defined as

$\mu_{r}^{\prime}=E\left(x^{r}\right)=\int_{x=0}^{\infty} x^{r} f(x, \alpha)=\alpha^{r} \Gamma(1-r)$.

This integral exist only for $r<1$.

\subsection{Inverse Moments}

The problem of inverse moments are arises naturally, when we are not in position to obtain the explicit expression for the descriptive statistics such as mean, variance etc. in inverted family of distributions, then in that situation the method of inverse moments can be thoughtof. For more details about inverse moment see [26]. In above subsection, we see that the moment for IED does not exist for $r>1$, therefore, if we evaluate inverse moment i.e.

$$
\begin{aligned}
E\left(\frac{1}{x^{r}}\right) & =\int_{x=0}^{\infty} \frac{1}{x^{r}} f(x, \alpha) d x \\
& =\frac{\Gamma(1+r)}{\alpha^{r}}
\end{aligned}
$$

Thus, from the above expression, one can obtain the first four inverse moments about origin by considering $r=$ $1,2, \cdots, 4$. 


\subsection{Inverse Moment Generating Function (IMGF)}

In statistical literature, several indirect methods are available to generate moments. Method of moment generating function (m.g.f.) is one of them. It is very useful method to obtain the moments by generating method. Generally, in inverse family of distribution the method of IMGF can be more appropriate and useful. IMGF in the case of considered model is obtained as;

$$
\begin{aligned}
I M_{X-1}(t) & =E\left(e^{t / x}\right) \\
= & \int_{x=0}^{\infty} e^{t / x} f(x \mid \alpha) d x \\
= & \frac{\alpha}{\alpha-t} \quad ; \alpha>t
\end{aligned}
$$

From the above expression of IMGF the first four moments are obtained by differentiating $I M_{X^{-1}}(t)$ with respect to $t$ and putting $t=0$ for respective choice of moment.

\subsection{Inverse Characteristics Function}

Let $X$ is a random variable (r.v.) with probability density function (1) and cumulative distribution function (2). Govindarajulu derived the mathematical properties related to the basic distribution such as binomial, Poisons, negative binomial e.t.c. see $[27,28]$. Then, the inverse characteristic function $\Phi_{X^{-1}}(t)$ of r.v $X$ is defined as;

$$
\begin{aligned}
\Phi_{X^{-1}}(t) & =E\left(e^{i t / x}\right) \\
= & \int_{x=0}^{\infty} e^{i t / x} f(x \mid \alpha) d x \\
= & \frac{\alpha}{\alpha-i t}
\end{aligned}
$$

\subsection{Quantile Function}

Let $X$ be a random variable with probability density function (1), then the quantile function $Q_{\phi}$ is defined by $F\left(Q_{\phi}\right)=\frac{\phi}{4}$ is the root of the equation i.e.

$$
\begin{aligned}
e^{-\alpha / Q_{\phi}} & =\frac{\phi}{4} \\
\Rightarrow Q_{\phi} & =\left(\frac{\alpha}{\ln 4-\ln \phi}\right) \quad ; \phi=1,2 \& 3
\end{aligned}
$$

The quartiles of IED model are computed for respective values of $\phi$ and are given as follows;

First Quartile: $Q_{1}=\left(\frac{\alpha}{2 \ln 2}\right)$

Second Quartile: $Q_{2}=\left(\frac{\alpha}{\ln 2}\right)$

Third Quartile: $Q_{3}=\left(\frac{\alpha}{\ln 4-\ln 3}\right)$

\subsection{Mode}

If $X$ be a random variable observed from density function (1). Then, mode of the above considered model can be obtained by differentiating the pdf (1) w.r.t. X and equating to zero, i.e. the mode is solution of the equation $f^{\prime}(x)=0$. Thus we have,

$f^{\prime}(x)=e^{-\alpha / x}\left\{\frac{\alpha}{x^{4}}-\frac{1}{x}\right\}=0$

Therefore, the mode $M$ of the inverted exponential density function $\mathrm{f}(\mathrm{x})$ is given by $M=\alpha^{1 / 3}$ 


\subsection{Harmonic Mean}

Let $X$ be a random variable observed from inverted exponential distribution having pdf (1). Then, the harmonic mean of a r.v. $X$ is defined as;

$$
\frac{1}{H}=\int_{x}\left(\frac{1}{x}\right) f(x, \alpha) d x
$$

After simplifying the above integral, the harmonic mean of the considered distribution is obtained as $H=\alpha$

\subsection{Order Statistics}

In this subsection, we derived the mathematical expression for $r^{t h}$ order statistics. Let us consider $x_{(1)}, x_{(2)}, \cdots, x_{(n)}$ are the $n$ ordered random sample observed from (1). Then the p.d.f. of $r^{\text {th }}$ order statistics is given by

$f_{r}\left(x_{(r)}, \alpha\right)=\frac{n !}{(r) !(n-r) !}\left[F\left(x_{(r)}\right)\right]^{r-1}\left[1-F\left(x_{(r)}\right)\right]^{n-r} f\left(x_{(r)}, \alpha\right)$

After using the expression (1) and (2) for $x_{(r)}$ in above equation we get the expression for $r^{\text {th }}$ order statistics. i.e.

$f_{r}\left(x_{(r)}, \alpha\right)=\frac{1}{B(r, n-r+1)}\left[\frac{\alpha}{x_{(r)}^{2}} e^{-r \alpha / x_{(r)}}\right]\left[1-e^{-r \alpha / x_{(r)}}\right]^{n-r}$

This is the required expression for $r-t h$ order statistics. If we put $r=1, n$, then, we get the distribution of $x_{(1)}=\min \left(x_{(1)}, x_{(2)}, \cdots, x_{(n)}\right)$ and $x_{(n)}=\max \left(x_{(1)}, x_{(2)}, \cdots, x_{(n)}\right)$ respectively, are given by;

$f_{1}\left(x_{(1)}, \alpha\right)=\frac{n \alpha}{x_{(1)}^{2}} e^{-\alpha / x_{(1)}}\left[1-e^{-\alpha / x_{(1)}}\right]^{n-1}$

$f_{n}\left(x_{(n)}, \alpha\right)=\frac{n \alpha}{x_{(n)}^{2}} e^{-n \alpha / x_{(n)}}$

\subsection{Stochastic Ordering}

The concept of stochastic ordering are frequently used to show the ordering mechanism in life time distributions. For more detail about stochastic ordering, see [4]. A random variable $X$ is said to be stochastically greater $\left(Y \leq_{s t} X\right)$ than $Y$ if $F_{X}(x) \leq F_{Y}(x)$ for all $x$. In the similar way, $X$ is said to be stochastically greater $\left(X \leq_{s t} Y\right)$ than $Y$ in the

- stochastic order $\left(X \leq_{s t} Y\right)$ if $F_{X}(x) \geq F_{Y}(x)$ for all $\mathrm{x}$.

- hazard rate order $\left(X \leq_{h r} Y\right)$ if $h_{X}(x) \geq h_{Y}(x)$ for all $\mathrm{x}$.

- mean residual life order $\left(X \leq_{m r l} Y\right)$ if $m_{X}(x) \geq m_{Y}(x)$ for all $\mathrm{x}$.

- likelihood ratio order $\left(X \leq_{l r} Y\right)$ if $\left(\frac{f_{X}(x)}{f_{Y}(x)}\right)$ decreases in $\mathrm{x}$.

From the above relations, we analysed that;

$$
\left(X \leq_{l r} Y\right) \Rightarrow\left(X \leq_{h r} Y\right) \Downarrow\left(X \leq_{s t} Y\right) \Rightarrow\left(X \leq_{m r l} Y\right)
$$

The inverted exponential distributions are ordered with respect to the strongest likelihood ratio ordering as shown in the following lemma.

Lemma: Let $X \sim \operatorname{IED}\left(\alpha_{1}\right)$ and $Y \sim \operatorname{IED}\left(\alpha_{2}\right)$. If $\alpha_{1}>\alpha_{2}$, then $\left(X \leq_{l r} Y\right)$ and hence $\left(X \leq_{h r} Y\right),\left(X \leq_{m r l} Y\right)$ and $\left(X \leq_{s t} Y\right)$. 
Proof: According to the definition of likelihood ratio order first we obtain the ratio $\left[\frac{f_{X}(x)}{f_{Y}(x)}\right]$ i.e.

$$
\begin{aligned}
\psi=\frac{f_{X}(x)}{f_{Y}(x)} & =\frac{\frac{\alpha_{1}}{x^{2}} e^{-\alpha_{1} / x}}{\frac{\alpha_{2}}{x^{2}} e^{-\alpha_{2} / x}} \\
& =\frac{\alpha_{1}}{\alpha_{2}} e^{-\left(\alpha_{1}-\alpha_{2}\right) / x}
\end{aligned}
$$

Therefore,

$\psi^{\prime}=\frac{\alpha_{1}}{\alpha_{2}} \frac{\left(\alpha_{1}-\alpha_{2}\right)}{x^{2}} e^{-\left(\alpha_{1}-\alpha_{2}\right) / x}$

from above equation, we observed that, if $\alpha_{1}>\alpha_{2} \Rightarrow \psi^{\prime}>0$, hence $\left(X \leq_{l r} Y\right)$. The remaining statements follow from the above relationship.

\subsection{Entropy Measurement}

Entropy measures provide important tools to indicate variety in distributions at particular moments in time and to analysis evolutionary processes over time. This measures the variation of the uncertainty in the distribution of a random variable $X$. For a probability distribution, [3] gave an expression of the entropy function, so called Renyi entropy and is defined by

$\zeta(\mu)=\frac{1}{1-\mu} \ln \left\{\int_{x} f^{\mu}(x, \alpha) d x\right\}$

where, $\mu>0$ and $\mu \neq 1$. For the inverted exponential distribution we obtained that

$\zeta(\mu)=\frac{1}{1-\mu} \ln \left\{\int_{x} \frac{\alpha^{\mu}}{x^{2 \mu}} e^{-\alpha \mu / x} d x\right\}$.

After simplification we get;

$\zeta(\mu)=\ln \alpha+\left(\frac{1-2 \mu}{1-\mu}\right) \ln \mu+\frac{\ln \Gamma(2 \mu-1)}{1-\mu}$

\section{Stress-Strength reliability}

Let $X$ and $Y$ are the independent stress and strength random variables observed from inverted exponential distribution with parameters $\alpha_{1}$ and $\alpha_{2}$ respectively. Then, the stress-strength reliability $R$ is calculated as;

$$
\begin{aligned}
R=P(X>Y) & =\int_{x=0}^{\infty}\left\{\int_{y=0}^{x} f\left(y, \alpha_{2}\right) d y\right\} f\left(x, \alpha_{1}\right) d x \\
& =\int_{x=0}^{\infty} f\left(x, \alpha_{1}\right) F\left(x, \alpha_{2}\right) d x \\
& =\int_{0}^{\infty} \frac{\alpha_{1}}{x^{2}} e^{-\alpha_{1} / x} e^{-\alpha_{2} / x} d x \\
& =\frac{\alpha_{1}}{\alpha_{1}+\alpha_{2}}
\end{aligned}
$$

From the above, we observed that the stress-strength reliability $R$ is the function of stress-strength parameter $\alpha_{1}$ and $\alpha_{2}$ respectively. Therefore, for maximum likelihood estimate (MLE) of $R$, we need to obtain the MLEs of $\alpha_{1}$ and $\alpha_{2}$. 


\section{Maximum likelihood estimation}

Let us consider $x_{1}, x_{2}, \cdots, x_{n}$ and $y_{1}, y_{2}, \cdots, y_{m}$ are independent random samples from $\operatorname{IED}\left(\alpha_{1}\right)$ and $\operatorname{IED}\left(\alpha_{2}\right)$, respectively. Then, the likelihood function of $\alpha_{1}$ and $\alpha_{2}$ are written as

$L\left(\alpha_{1}, \alpha_{2} \mid \underline{\mathrm{x}}, \mathrm{y}\right)=\frac{\alpha_{1}^{n} e^{-\alpha_{1} s_{1}} \alpha_{2}^{m} e^{-\alpha_{2} s_{2}}}{\prod_{i=1}^{n} x_{i}^{2} \prod_{j=1}^{m} y_{j}^{2}}$

where, $s_{1}=\sum_{i=1}^{n} \frac{1}{x_{i}}$ and $s_{2}=\sum_{j=1}^{m} \frac{1}{y_{j}}$, then, the log-likelihood equation is written as:

$\ln L\left(\alpha_{1}, \alpha_{2}\right)=n \ln \alpha_{1}+m \ln \alpha_{2}-\alpha_{1} s_{1}-\alpha_{2} s_{2}-2 \sum_{i=1}^{n} \ln x_{i}-2 \sum_{j=1}^{m} \ln y_{j}$

The MLE of $\alpha_{1}$ and $\alpha_{2}$, say $\hat{\alpha_{1}}$ and $\hat{\alpha_{2}}$ respectively can be obtained by solving the equations;

$$
\frac{\partial \ln L}{\partial \alpha_{1}}=0, \frac{\partial \ln L}{\partial \alpha_{2}}=0, \text { which gives, } \hat{\alpha_{1}}=\frac{n}{s_{1}} \text { and } \hat{\alpha_{2}}=\frac{m}{s_{2}}
$$

Hence, using the invariance properties of MLEs, the MLE of the parameter $R$ is given by;

$\hat{R}_{M L}=\frac{\hat{\alpha}_{1}}{\hat{\alpha}_{1}+\hat{\alpha}_{2}}$

\section{Asymptotic distribution for $\mathrm{R}$}

In this section, we derived the asymptotic distribution of $\hat{R}$. Further, we have also constructed $95 \%$ asymptotic confidence interval based on its asymptotic distribution. Using large theory concept as we know that,

$\sqrt{n}\left(\hat{\alpha}_{k}-\alpha_{k}\right) \rightarrow N\left(0, \frac{1}{\hat{\sigma}_{k}{ }_{k}}\right)$

where, $\hat{\sigma}_{k}^{2}=\alpha_{k}^{2}$ and $k=1,2$. Therefore, the asymptotic $100(1-\theta) \%$ confidence intervals for $\alpha_{1}$ and $\alpha_{2}$ are constructed as;

$\hat{\alpha}_{k} \mp Z_{\theta / 2} \hat{\alpha}_{k}$

where, $z_{\theta / 2}$ is the upper $(\theta / 2)$ th percentile of a standard normal variable. Similarly, the asymptotic distribution of stress-strength reliability as $n \rightarrow \infty$ and $m \rightarrow \infty$ is given by

$\frac{\hat{R}_{M L}-R}{\sqrt{\frac{R^{\prime}\left(\alpha_{1}\right)}{n \sigma_{1}^{2}}+\frac{R^{\prime}\left(\alpha_{2}\right)}{n \sigma_{2}^{2}}}} \rightarrow N(0,1)$

where,

$R^{\prime}\left(\alpha_{1}\right)=\frac{\alpha_{2}}{\left(\alpha_{1}+\alpha_{2}\right)^{2}}$ and $R^{\prime}\left(\alpha_{2}\right)=-\frac{\alpha_{1}}{\left(\alpha_{1}+\alpha_{2}\right)^{2}}$.

Although, $\hat{R}_{M L}$ can be obtained in explicit form, the exact distribution of $\hat{R}_{M L}$ is difficult to obtain. Due to this reason, to construct the confidence interval of $R$ we mainly consider the confidence interval based on the asymptotic distribution of $\hat{R}_{M L}$. Thus, the $100(1-\theta) \%$ asymptotic confidence interval for the parameter $R$ is calculated as;

$\left\{\hat{R}_{M L} \mp Z_{\theta / 2} \sqrt{\frac{R^{\prime}\left(\alpha_{1}\right)}{n \sigma_{1}^{2}}+\frac{R^{\prime}\left(\alpha_{2}\right)}{n \sigma_{2}^{2}}}\right\}$. 


\section{Bayes estimation}

In this section, we have developed the Bayesian estimation procedure for the estimation of stress-strength reliability $R$ from inverted exponential distribution assuming independent gamma priors for the unknown model parameters. Thus, the priors of $\alpha_{1}$ and $\alpha_{2}$ are given as:

$$
\pi\left(\alpha_{1}\right)=\frac{b^{a}}{\Gamma(a)} \alpha_{1}^{a-1} e^{-b \alpha_{1}}
$$

and

$$
\pi\left(\alpha_{2}\right)=\frac{d^{c}}{\Gamma(c)} \alpha_{2}^{c-1} e^{-d \alpha_{2}}
$$

Therefore, using (15) and mentioned prior distribution, the joint posterior distribution of $\alpha_{1}$ and $\alpha_{2}$ given data is

$$
\begin{aligned}
\pi\left(\alpha_{1}, \alpha_{2} \mid \underline{\mathrm{x}}, \underline{\mathrm{y}}\right) & =\frac{\alpha_{1}^{n+a-1} \alpha_{2}^{m+c-1} \exp \left[-\alpha_{1}\left(b+s_{1}\right)-\alpha_{2}\left(d+s_{2}\right)\right]}{\int_{\alpha_{1}, \alpha_{2}} \alpha_{1}^{n+a-1} \alpha_{2}^{m+c-1} \exp \left[-\alpha_{1}\left(b+s_{1}\right)-\alpha_{2}\left(d+s_{2}\right)\right] d \alpha_{1} d \alpha_{2}} \\
& =\frac{\left(b+s_{1}\right)^{n+a}\left(d+s_{2}\right)^{m+c}}{\Gamma(n+a) \Gamma(m+c)} \alpha_{1}^{n+a-1} \alpha_{2}^{m+c-1} \exp \left[-\alpha_{1}\left(b+s_{1}\right)-\alpha_{2}\left(d+s_{2}\right)\right] \\
& =\operatorname{Gamma}\left(n+a, b+s_{1}\right) * \operatorname{Gamma}\left(m+c, d+s_{2}\right)
\end{aligned}
$$

Since $\alpha_{1}$ and $\alpha_{2}$ are independent. Therefore, the equation (25) implies that, the posterior distribution of $\alpha_{1}$ and $\alpha_{2}$ are

$\pi\left(\alpha_{1} \mid \underline{\mathrm{x}}\right) \sim \operatorname{Gamma}\left(n+a, b+s_{1}\right)$

and

$\pi\left(\alpha_{2} \mid \mathrm{y}\right) \sim \operatorname{Gamma}\left(m+c, d+s_{2}\right)$

Now, for obtaining the Bayes estimate of the stress-strength parameter $R$, we derived the posterior distribution of $R$. Since $\alpha_{1}$ and $\alpha_{2}$ are independent and using (26) and (27), the posterior distribution of $R$ becomes:

$\pi(r \mid \underline{\mathrm{x}}, \underline{\mathrm{y}})=\left\{\frac{\left(b+s_{1}\right)^{n+a}\left(d+s_{2}\right)^{m+c}}{\operatorname{Bet} a(n+a, m+c)}\right\}\left[\frac{r^{n+a}(1-r)^{m+c-1}}{\left[r\left(b+s_{1}\right)+(1-r)\left(d+s_{2}\right)\right]^{n+m+a+c}}\right] \quad ; 0 \leq r \leq 1$

To obtain the estimates of $R$ under Bayesian paradigm, we must associate any loss function. Here, we used two loss functions namely squared error loss function (SELF) and linex loss function (LLF). Thus, the mathematical formulation of the SELF is given as;

$L(\theta, \hat{\theta})=(\theta-\hat{\theta})^{2}$

where, $\hat{\theta}$ is the estimated values of $\theta$. The beauty of SELF is that it equally associates the over as well as under estimation. Under this loss function, the Bayes estimate of $R$ is nothing but mean of the posterior distribution of $R$, which is given in following equation.

$$
\begin{aligned}
\hat{R}_{B S} & =\int_{r=0}^{1} r \pi(r \mid \underline{\mathrm{x}}, \underline{\mathrm{y}}) d r \\
& =\int_{r=0}^{1}\left\{\frac{\left(b+s_{1}\right)^{n+a}\left(d+s_{2}\right)^{m+c}}{\operatorname{Beta}(n+a, m+c)} \frac{r^{n+a}(1-r)^{m+c-1}}{\left[r\left(b+s_{1}\right)+(1-r)\left(d+s_{2}\right)\right]^{n+m+a+c}}\right\} d r
\end{aligned}
$$

and the LLF is defined as;

$L(\theta, \hat{\theta})=e^{-\delta \Delta}-\delta \Delta-1$

where, $\delta$ is loss parameter, which reflects the departure from symmetry and $\Delta=(\theta-\hat{\theta})$. For more detail about this loss function you may see [25]. Using LLF the Bayes estimates of the parameter $R$ is evaluated by the following equation

$\hat{R}_{B L}=-\frac{1}{\delta} \ln E(\exp (-\delta r))$ 
The positive (negative) values of $\delta$ reflected that over (under) estimation is more serious than under estimation and vice versa. Here, we took $\delta=0.5$ (over estimation) and $\delta=-0.5$ (under estimation). Therefore, the corresponding expressions $\hat{R}_{B L}$ (for $\delta=0.5$ ), $\hat{R}_{B L 1}$ (for $\delta=-0.5$ ) are;

$$
\begin{aligned}
& \hat{R}_{B L}=\int_{r=0}^{1}\left\{\frac{\left(b+s_{1}\right)^{n+a-1}\left(d+s_{2}\right)^{m+c}}{\operatorname{Beta}(n+a, m+c)} \frac{e^{-0.5 r} r^{n+a}(1-r)^{m+c-1}}{\left[r\left(b+s_{1}\right)+(1-r)\left(d+s_{2}\right)\right]^{n+m+a+c}}\right\} d r \\
& \hat{R}_{B L 1}=\int_{r=0}^{1}\left\{\frac{\left(b+s_{1}\right)^{n+a-1}\left(d+s_{2}\right)^{m+c}}{\operatorname{Beta}(n+a, m+c)} \frac{e^{0.5 r} r^{n+a}(1-r)^{m+c-1}}{\left[r\left(b+s_{1}\right)+(1-r)\left(d+s_{2}\right)\right]^{n+m+a+c}}\right\} d r .
\end{aligned}
$$

The analytical solution of the above equations (30), (33) and (34) are not possible due to its mathematical mess. Therefore, we may propose the use of any approximation technique to solve such integrals. Here, we suggest the use of Gauss quadrature method of approximation.

\section{Real data application}

In this section, we proposed the real life applicability of the proposed methodology in previous sections. For this purpose, we have considered two data sets, which are initially proposed by [21]. The data represents the patients of two groups suffering from head and neck cancer disease. Recently [2] is also modelled this data using log-normal model and discussed its posterior based inferences. The data set of first group represents the survival times of 51 head and neck cancer patients treated with radiotherapy whereas the other group of data set represents the survival times of 45 head and neck cancer patients treated with combined radiotherapy and chemotherapy. The data sets are as follows:

Data (X): $6.53,7,10.42,14.48,16.10,22.70,34,41.55,42,45.28,49.40,53.62,63,64,83,84,91,108,112,129,133$, $133,139,140,140,146,149,154,157,160,160,165,146,149,154,157,160,160,165,173,176,218,225,241,248,273$, $277,297,405,417,420,440,523,583,594,1101,1146,1417$

Data (Y): $12.20,23.56,23.74,25.87,31.98,37,41.35,47.38,55.46,58.36,63.47,68.46,78.26,74.47,81,43,84,92$, $94,110,112,119,127,130,133,140,146,155,159,173,179,194,195,209,249,281,319,339,432,469,519,633,725$, 817,1776

To check the validity of the considered model for the above data sets $X$ and $Y$, we discussed about the various statistical tools such as Kolmogrov-Smirnov (K-S) test, log-likelihood criterion, Aikaiki information criterion (AIC) and Bayesian information criterion (BIC). The applicability of this model for the above data sets over inverse Rayleigh distribution (IRD), generalized inverted exponential distribution (GIED) have discussed and it is found that the considered model is quite flexible than the other two, see Table (4). Further, we also plotted the empirical cumulative distribution plot and Q-Q plot for both data set, see Figures $1,2 \& 3$. The maximum likelihood estimate and Bayes estimates of the parameters $\left(\alpha_{1}, \alpha_{2}\right)$ and stress strength parameter $R$ are obtained as $(59.12593,75.37942)$ and $\left(R_{M L}=0.4395805, R_{B S}=0.5141707\right)$ respectively. On the consideration of the above data set, the usefulness of the considered model over other distribution under the above criterion following ordering have been observed.

\section{For data set-I:}

- On the basis of AIC, Log-likelihood and K-S distance;

$$
\text { Best } \quad G I E D \rightarrow I E D \rightarrow I R D(\text { Worst })
$$

- On the basis of Bayesian information criterion;

$$
\text { Best } I E D \rightarrow G I E D \rightarrow I R D(\text { Worst })
$$

\section{For data set-II:}

- On the basis of AIC, BIC and K-S distance;

$$
\text { Best } I E D \rightarrow G I E D \rightarrow I R D \text { (Worst) }
$$


Figure 1: ECDF plot for data set-I

\section{Emperical cumulative distribution plot for Data set-I}

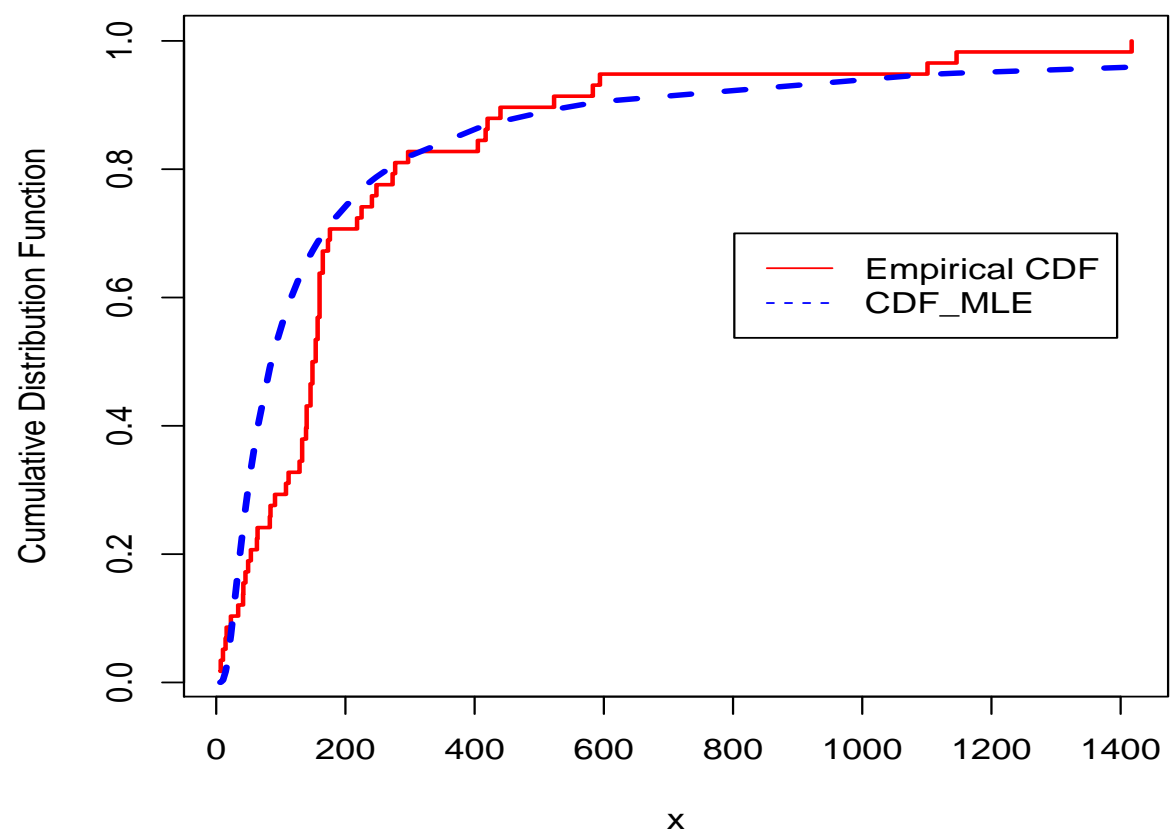

Figure 2: ECDF plot for data set-II

Emperical cumulative distribution plot for Data set-II

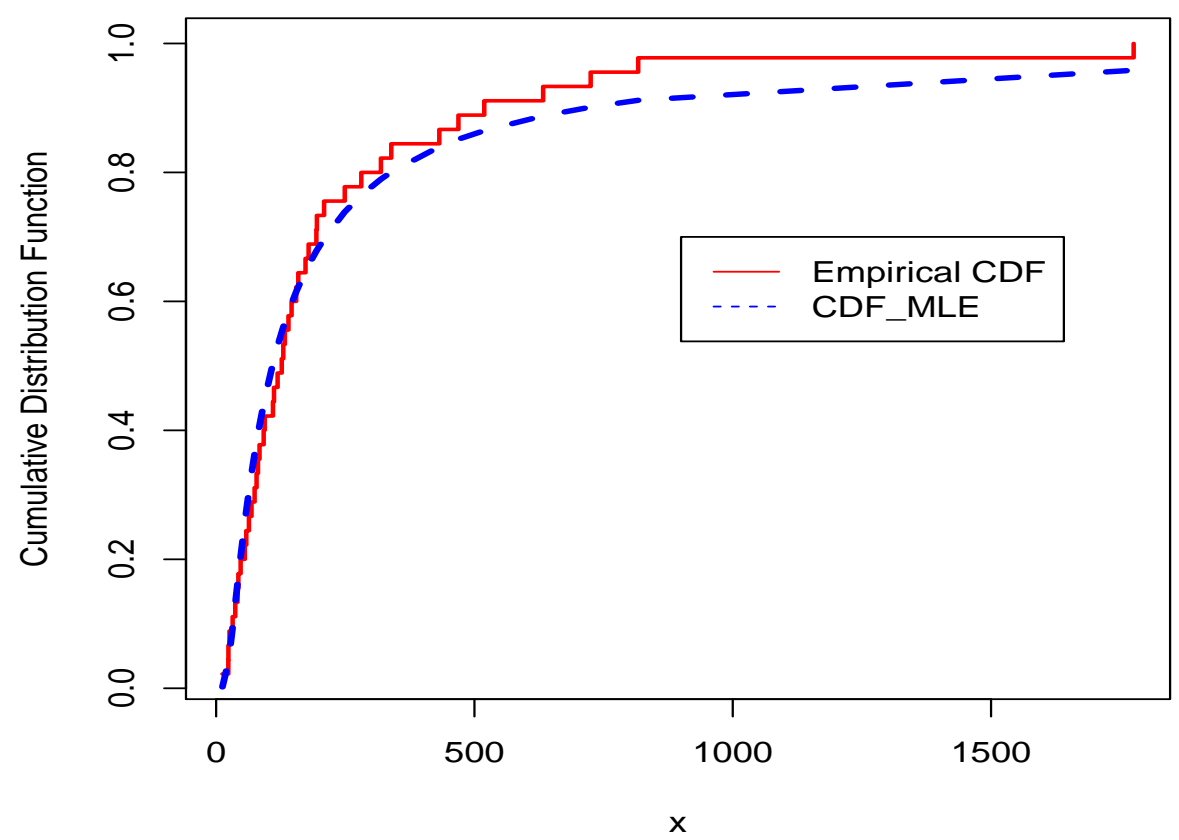


Figure 3: QQ-plots regarding fitting of the model based on data set-I and II
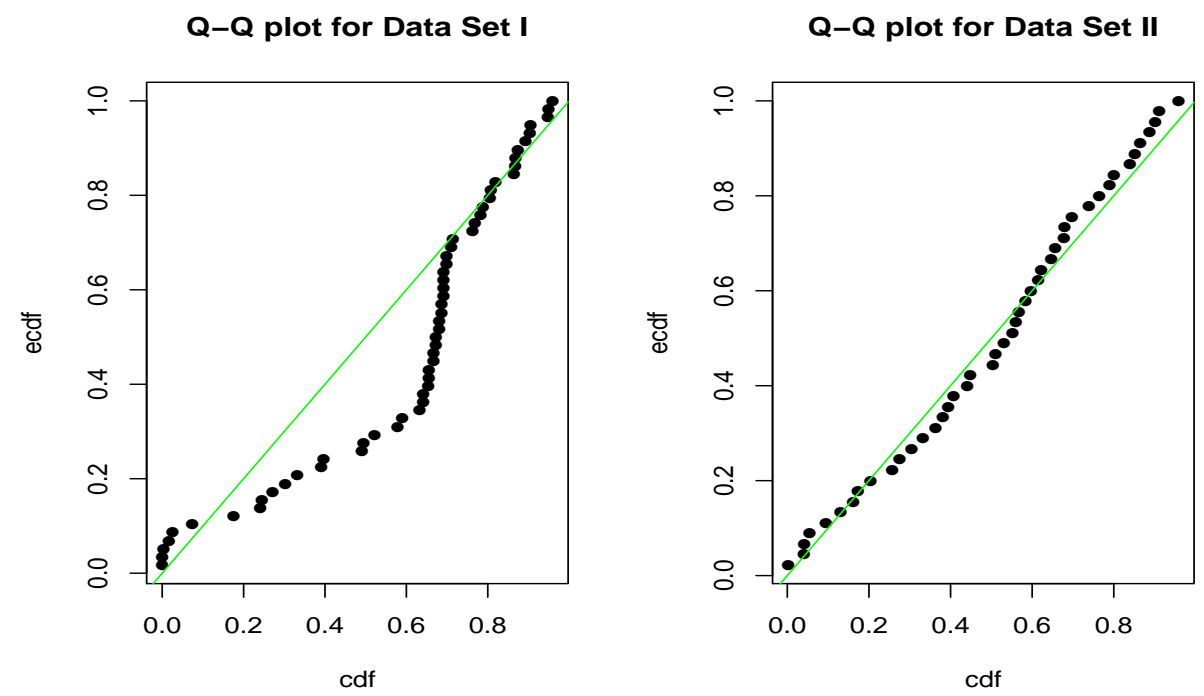

- On the basis of log-likelihood criterion;

$$
\text { Best } \quad G I E D \rightarrow I E D \rightarrow I R D(\text { Worst })
$$

Thus, after observing the above pattern, we can recommend that one can also use inverted exponential distribution for modelling of the considered data sets. The plots of estimated probability density function, survival function and hazard function are also given, see Figure 4 and 5 respectively.

\section{Numerical study}

In this section, we investigate the performances of the estimators through Monte Carlo Simulation. The simulation study is carried out for different variation of $n, m$ and model parameters $\alpha_{1}, \alpha_{2}$ respectively. The performances of the MLEs and the Bayes estimates are compared in terms of mean squares errors (MSEs). The Bayes estimates are computed under both informative and non-informative priors. For non-informative prior, we took $a=b=c=d=0$ and call it Bayes 0. For informative priors, the values of hyper parameters are chosen in such a way that the prior variance is very small (0.5) and large (10) i.e. our prior belief is much closer to the true or apart from true belief. Prior with small variance is called as Bayes 1 and prior with large variance is called as Bayes 2. All the simulation results are based on 10000 replications and presented in Tables 1,2 and 3. From this extensive study we observed that,

- From the Tables of simulation, we noticed that the performances of the Bayes estimators are better than the corresponding maximum likelihood estimators in all considered cases.

- The MSE's of the Bayes as well as maximum likelihood estimates of stress-strength parameter $R$ are decreases as sample sizes $(n, m)$ increases and similar trends have been observed when we fixed $n$ with varying $m$ and vice versa.

- Under informative prior (Bayes 1) the MSE's of the Bayes estimates of stress-strength parameter $R$ are smaller than the MSE's of the MLE's and the performances of the Bayes estimates and ML estimates are quite similar under Bayes 2 and Bayes 0, see Tables 1,2, and 3.

- From the Table 1, 2 and 3 we observed that as true values of $R$ is increases then the MSE's of the estimators are decreases. It means that the efficiency of the estimator $R$ will be maximum for higher value of the actual reliability.

- MSE's of the Bayes estimators under squared error loss function and linex loss function are quite similar. Therefore, both the losses can be used for the estimation of the parameter $R$. 
Figure 4: Estimated daensity, survival function and hazard function plots based on data set-I

Estimated density Plot for Data set-I

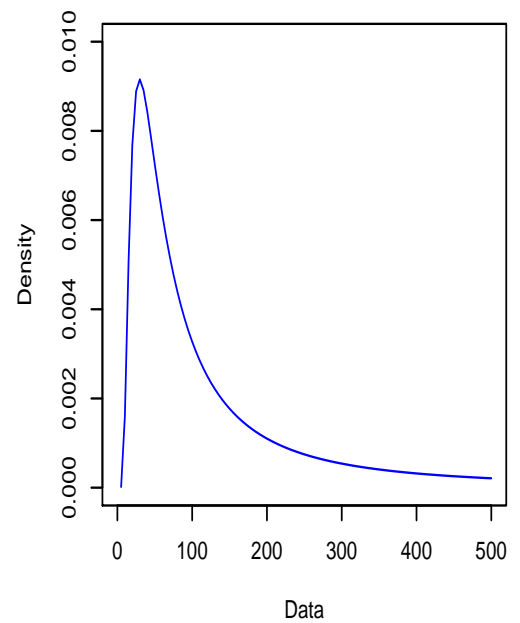

Estimated survival function for Data Set-I

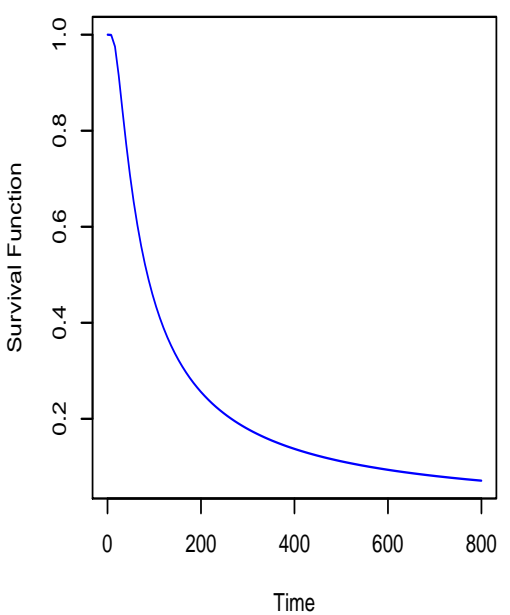

Estimated hazard function for Data Set-I

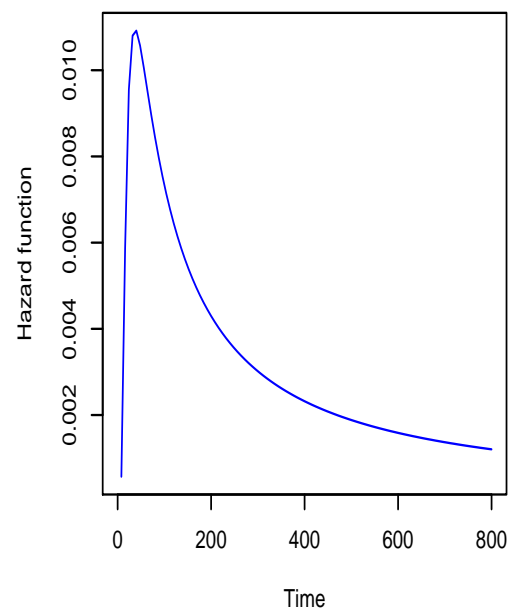

Figure 5: Estimated daensity, survival function and hazard function plots based on data set-II

Estimated density Plot for Data set-II

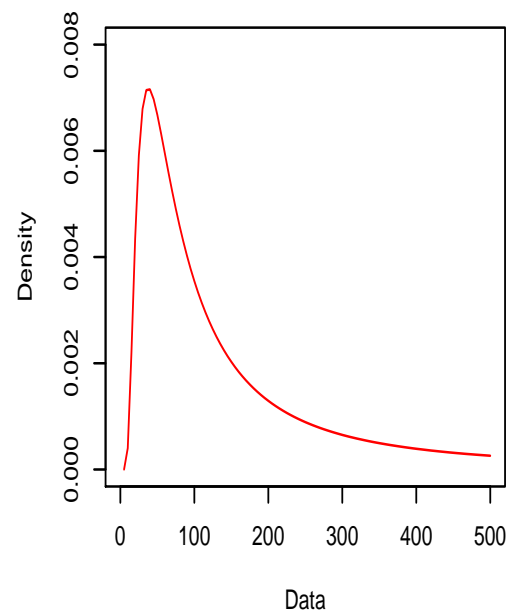

Estimated survival function for Data Set-II

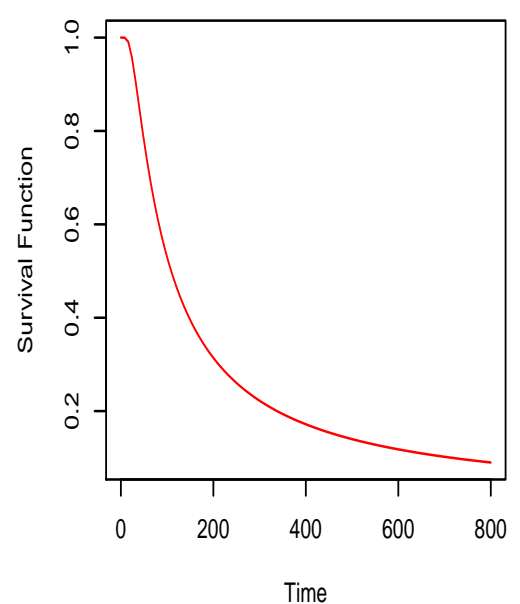

Estimated hazard function for Data Set-II

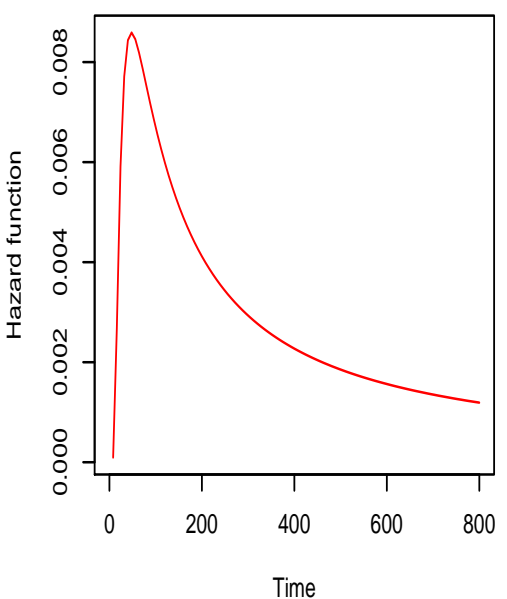




\section{Concluding remarks}

In this paper, we consider the estimation of the stress-strength parameter $R$ from inverted exponential distribution and derived its mathematical and statistical properties such as moments, inverse moment, inverse moment generating function, quantile, mode, stochastic ordering, entropy measurements and order statistics. The problem of estimating the stress-strength reliability $R=P[Y<X]$, where $X$ and $Y$ follow the inverted exponential distributions have been discussed by both classical and Bayesian methods of estimation. The asymptotic distribution of the parameter $R$ is also derived. The Bayes estimates of $R$ is carried out under both informative and non-informative priors using squared error and linex loss functions. From the simulation result, we concluded that Bayes estimators obtained under informative prior perform well and have smaller mean squared error as compared to that of obtained under the non-informative prior and maximum likelihood estimators. The performances of the estimators under linex loss function can also be appreciated under informative prior. Further, we have also considered two real data set to illustrate the applicability of the considered model in real life scenario and it is found that the considered model provides better fit to the given data set.

\section{Acknowledgements}

The authors thankful to the referees and editor for their valuable comments and suggestions for improving the manuscript. The third author (Abhimanyu Singh Yadav) thanks to UGC, New Delhi, India, for providing financial assistance.

\section{References}

[1] M. E. Ghitany, D. K. Al-Mutairi, and S. M. Aboukhamseen (2013), Estimation of the reliability of a stress-strength system from power lindley distributions. Communications in Statistics-Simulation and Computation (2013).

[2] Puja Makkar, Puneet K. Srivastava, R. S. Singh, and S. K. Upadhyay (2014), Bayesian survival analysis of head and neck cancer data using log normal model. Communications in Statistics - Theory and Methods 43,392-407.

[3] A. Renyi (1961), On measures of entropy and information, Proceedings of the 4th Berkeley Symposium on Mathematical Statistics and Probability. University of California Press, Berkeley, pp. 547-561.

[4] M. Shaked and J.G. Shanthikumar (1994), Stochastic orders and their applications, Boston: Academic Press.

[5] Bhupendra Singh and Puneet Kumar Gupta (2012), Load-sharing system model and its application to the real data set. Mathematics and Computers in Simulation 82, 1615-1629.

[6] Dey S. (2007). Inverted exponential distribution as a life distribution model from a Bayesian viewpoint. Data Sci. J. 6: 107-113.

[7] Lin C., Duran B. S. and Lewis T. O. (1989). Inverted gamma as a life distribution. Microelectron. Reliab. 29 (4):619-626.

[8] Prakash G. (2009). Some estimation procedures for the inverted exponential distribution. The South Pacific Journal of Natural Science, 27:71-78.

[9] Singh S. K., Singh U. and Kumar D.(2003). Bayes estimators of the reliability function and parameter of inverted exponential distribution using informative and non-informative priors. Journal of Statistical Computation and Simulation, 83(12): 2258-2269.

[10] Vikas Kumar Sharma, Sanjay Kumar Singh, and Umesh Singh (2014), A new upside-down bathtub shaped hazard rate model for survival data analysis. Applied Mathematics and Computation 239, 242-253.

[11] Ali, M., Pal, M., and Woo, J. (2010). Estimation of $\operatorname{Pr}(\mathrm{Y}<\mathrm{X})$ when $\mathrm{X}$ and $\mathrm{Y}$ belong to different distribution families. Journal of Probability and Statistical Science, 8, p. 35-48.

[12] Kundu, D. and Gupta, R. D. (2005). Estimation of $\mathrm{P}(\mathrm{Y}<\mathrm{X})$ for generalized exponential distribution. Metrika, 61, p. 291-308.

[13] Awad, A.M. and Gharraf, M.K. (1986). Estimation of $\mathrm{P}(\mathrm{Y}<\mathrm{X})$ in the Burr case: A comparative study. Communications in Statistics-Simulation 15:389-403. 
[14] Kundu, D. and Raqab, M.Z. (2009). Estimation of $\mathrm{R}=\mathrm{P}(\mathrm{Y}<\mathrm{X})$ for three-parameter Weibull distribution. Statistics and Probability Letters 79:1839-1846.

[15] Kundu, D. and Gupta, R.D. (2006). Estimation of $\mathrm{R}=\mathrm{P}[\mathrm{Y}<\mathrm{X}]$ for Weibull distributions. IEEE Transactions on Reliability 55:270-280.

[16] Pundir P. S., Singh B. P. and Maheshwari S (2014). On hybrid censored inverted exponential distribution. International Journal of Current Research, Vol. 6, Issue, 01, pp. 4539-4544.

[17] Singh S. K., Singh U., Vishwakarma P. K. and Yadav A. S (2014), Bayesian Reliability Estimation of Inverted Exponential Distribution Under Progressive Type-II Censored Data. J. Stat. Appl. Pro. 3, No. 3, 1-17.

[18] McCool, J.I., (1991). Inference on P. $(\mathrm{Y}<\mathrm{X})$ in the Weibull case. Communications in Statistics. Simulation and Computations 20, 129-148.

[19] Kotz, S., Lumelskii, Y. and Pensky, M., (2003). The Stress-Strength Model and Its Generalizations. World Scientific Press, Singapore.

[20] Sharma V. K., Singh S. K. , and Singh Umesh (2014), Estimation on System Reliability in Generalized Lindley StressStrength Model. J. Stat. Appl. Pro. 3, No. 1, 61-75.

[21] Efron, B. (1988). Logistic regression, survival analysis, and the Kaplan-Meier curve. J. Amer.Statist. Assoc. 83:414-425.

[22] Church, J.D. and Harris, B. (1970). The estimation of reliability from stress-strength relationships. Technometrics 12:49-54.

[23] Birnbaum, Z.W. (1956). On a use of the Mann-Whitney statistic. In: Proceedings of Third Berkeley Symposium on Mathematical Statistics and Probability, vol. 1, pp. 13-17, University of California Press, Berkeley, CA.

[24] Birnbaum, Z.W., McCarty, R.C. (1958). A distribution-free upper confidence bound for $P(Y<X)$, based on independent samples of X and Y. Annals of Mathematical Statistics 29:558-562.

[25] A. Zellner (1986). A Bayesian estimation and prediction using asymmetric loss function, Journal of American Statistical Association, 81, pp. 446-451.

[26] Zakkula Govindarajulu (1962). )Theory of inverse moments, AFOSR 62-72.

[27] David, F.N. and Johnson, N.L. (1957). Reciprocal Bernoulli and Poisson variables. Metron 18, 77-81.

[28] Zakkula Govindarajulu (1962). The Reciprocal of the decapitated negative binomial variable. To appear in the December issue of the Journal of the Amer. Statist. Assn. 57. 
Table 1: Average estimates (first row) and MSE's (second row) of the stress-strength reliability $(R=0.4)$ for different variation of $n, m$ when $\alpha_{1}=2$ and $\alpha_{2}=3$

\begin{tabular}{|c|c|c|c|c|c|c|c|c|c|c|}
\hline \multirow{2}{*}{$\mathrm{n}, \mathrm{m}$} & \multirow{2}{*}{$\begin{array}{l}\text { MLE } \\
\hat{R}_{M L}\end{array}$} & \multicolumn{3}{|c|}{ Bayes1 } & \multicolumn{3}{|c|}{ Bayes2 } & \multicolumn{3}{|c|}{ Bayes0 } \\
\hline & & $\hat{R}_{B S}$ & $\hat{R}_{B L}$ & $\hat{R}_{B L 1}$ & $\hat{R}_{B S}$ & $\hat{R}_{B L}$ & $\hat{R}_{B L 1}$ & $\hat{R}_{B S}$ & $\hat{R}_{B L}$ & $\hat{R}_{B L 1}$ \\
\hline \multirow{2}{*}{5,5} & 0.4097 & 0.4393 & 0.4345 & 0.4442 & 0.4167 & 0.4118 & 0.4216 & 0.4174 & 0.4125 & 0.4222 \\
\hline & 0.0220 & 0.0112 & 0.0107 & 0.0116 & 0.0182 & 0.0180 & 0.0184 & 0.0193 & 0.0190 & 0.0196 \\
\hline \multirow{2}{*}{10,10} & 0.4045 & 0.4231 & 0.4206 & 0.4257 & 0.4087 & 0.4061 & 0.4113 & 0.4106 & 0.4079 & 0.4132 \\
\hline & 0.0112 & 0.0078 & 0.0076 & 0.0080 & 0.0103 & 0.0102 & 0.0104 & 0.0104 & 0.0104 & 0.0106 \\
\hline \multirow{2}{*}{15,15} & 0.4031 & 0.4166 & 0.4147 & 0.4185 & 0.4067 & 0.4050 & 0.4084 & 0.4043 & 0.4026 & 0.4061 \\
\hline & 0.0075 & 0.0058 & 0.0058 & 0.0059 & 0.0071 & 0.0071 & 0.0072 & 0.0070 & 0.0070 & 0.0072 \\
\hline \multirow{2}{*}{20,20} & 0.4013 & 0.4119 & 0.4107 & 0.4131 & 0.4042 & 0.4027 & 0.4057 & 0.4047 & 0.4035 & 0.4060 \\
\hline & 0.0056 & 0.0046 & 0.0050 & 0.0049 & 0.0054 & 0.0058 & 0.0057 & 0.0054 & 0.0058 & 0.0057 \\
\hline \multirow{2}{*}{10,5} & 0.3965 & 0.4661 & 0.4622 & 0.4701 & 0.4158 & 0.4120 & 0.4196 & 0.4108 & 0.4071 & 0.4146 \\
\hline & 0.0160 & 0.0126 & 0.0121 & 0.0131 & 0.0142 & 0.0140 & 0.0144 & 0.0150 & 0.0149 & 0.0153 \\
\hline \multirow{2}{*}{10,15} & 0.4063 & 0.4065 & 0.4044 & 0.4087 & 0.4072 & 0.4050 & 0.4094 & 0.4074 & 0.4052 & 0.4095 \\
\hline & 0.0094 & 0.0064 & 0.0064 & 0.0065 & 0.0087 & 0.0087 & 0.0088 & 0.0087 & 0.0087 & 0.0088 \\
\hline \multirow{2}{*}{10,25} & 0.4107 & 0.3952 & 0.3934 & 0.3970 & 0.4047 & 0.4029 & 0.4066 & 0.4047 & 0.4029 & 0.4065 \\
\hline & 0.0082 & 0.0057 & 0.0056 & 0.0057 & 0.0074 & 0.0074 & 0.0075 & 0.0076 & 0.0076 & 0.0077 \\
\hline \multirow{2}{*}{10,35} & 0.4117 & 0.3894 & 0.3877 & 0.3910 & 0.4048 & 0.4030 & 0.4065 & 0.4064 & 0.4047 & 0.4081 \\
\hline & 0.0076 & 0.0053 & 0.0053 & 0.0053 & 0.0067 & 0.0067 & 0.0068 & 0.0070 & 0.0070 & 0.0071 \\
\hline \multirow{2}{*}{10,45} & 0.4119 & 0.3859 & 0.3842 & 0.3875 & 0.4055 & 0.4037 & 0.4072 & 0.4047 & 0.4030 & 0.4064 \\
\hline & 0.0074 & 0.0052 & 0.0053 & 0.0052 & 0.0067 & 0.0067 & 0.0068 & 0.0066 & 0.0066 & 0.0067 \\
\hline \multirow{2}{*}{10,75} & 0.4139 & 0.3823 & 0.3808 & 0.3838 & 0.4036 & 0.4020 & 0.4052 & 0.4053 & 0.4039 & 0.4067 \\
\hline & 0.0070 & 0.0049 & 0.0051 & 0.0050 & 0.0063 & 0.0063 & 0.0064 & 0.0064 & 0.0064 & 0.0065 \\
\hline \multirow{2}{*}{10,95} & 0.4137 & 0.3804 & 0.3789 & 0.3818 & 0.4034 & 0.4018 & 0.4050 & 0.4049 & 0.4035 & 0.4064 \\
\hline & 0.0067 & 0.0048 & 0.0050 & 0.0048 & 0.0062 & 0.0063 & 0.0063 & 0.0062 & 0.0063 & 0.0063 \\
\hline \multirow{2}{*}{5,10} & 0.4181 & 0.3977 & 0.3941 & 0.4013 & 0.4114 & 0.4077 & 0.4151 & 0.4120 & 0.4083 & 0.4157 \\
\hline & 0.0175 & 0.0085 & 0.0084 & 0.0085 & 0.0144 & 0.0142 & 0.0146 & 0.0152 & 0.0150 & 0.0154 \\
\hline \multirow{2}{*}{25,10} & 0.3954 & 0.4389 & 0.4368 & 0.4409 & 0.4080 & 0.4064 & 0.4098 & 0.4060 & 0.4042 & 0.4079 \\
\hline & 0.0079 & 0.0071 & 0.0069 & 0.0073 & 0.0073 & 0.0073 & 0.0076 & 0.0074 & 0.0075 & 0.0077 \\
\hline \multirow{2}{*}{10,15} & 0.4082 & 0.4081 & 0.4060 & 0.4102 & 0.4082 & 0.4060 & 0.4105 & 0.4068 & 0.4046 & 0.4089 \\
\hline & 0.0097 & 0.0066 & 0.0065 & 0.0067 & 0.0090 & 0.0089 & 0.0091 & 0.0085 & 0.0085 & 0.0087 \\
\hline \multirow{2}{*}{15,15} & 0.4031 & 0.4166 & 0.4146 & 0.4185 & 0.4068 & 0.4050 & 0.4086 & 0.4052 & 0.4035 & 0.4069 \\
\hline & 0.0074 & 0.0058 & 0.0058 & 0.0059 & 0.0070 & 0.0070 & 0.0071 & 0.0071 & 0.0070 & 0.0072 \\
\hline \multirow{2}{*}{20,15} & 0.4008 & 0.4210 & 0.4194 & 0.4226 & 0.4066 & 0.4050 & 0.4083 & 0.4061 & 0.4046 & 0.4076 \\
\hline & 0.0066 & 0.0056 & 0.0056 & 0.0057 & 0.0063 & 0.0064 & 0.0065 & 0.0064 & 0.0064 & 0.0066 \\
\hline \multirow{2}{*}{25,15} & 0.4010 & 0.4251 & 0.4237 & 0.4266 & 0.4059 & 0.4043 & 0.4074 & 0.4055 & 0.4040 & 0.4070 \\
\hline & 0.0060 & 0.0054 & 0.0056 & 0.0056 & 0.0057 & 0.0061 & 0.0059 & 0.0058 & 0.0062 & 0.0061 \\
\hline
\end{tabular}

Table 2: Average estimates (first row) and MSE's (second row) of the stress-strength reliability $R$ for different variation of $n, m$ when $\alpha_{1}=3$ and $\alpha_{2}=2$, when $R=0.5$

\begin{tabular}{ccccccccccc}
\hline \multirow{2}{*}{$\mathrm{n}, \mathrm{m}$} & MLE & \multicolumn{3}{c}{ Bayes1 } & \multicolumn{3}{c}{ Bayes2 } & \multicolumn{3}{c}{ Bayes0 } \\
\cline { 2 - 11 } & $\hat{R}_{M L}$ & $\hat{R}_{B S}$ & $\hat{R}_{B L}$ & $\hat{R}_{B L 1}$ & $\hat{R}_{B S}$ & $\hat{R}_{B L}$ & $\hat{R}_{B L 1}$ & $\hat{R}_{B S}$ & $\hat{R}_{B L}$ & $\hat{R}_{B L 1}$ \\
\hline \multirow{2}{*}{5,5} & 0.5039 & 0.5028 & 0.4979 & 0.5077 & 0.5014 & 0.4963 & 0.5065 & 0.4965 & 0.4915 & 0.5015 \\
& 0.0233 & 0.0099 & 0.0099 & 0.0099 & 0.0183 & 0.0183 & 0.0183 & 0.0187 & 0.0188 & 0.0187 \\
\multirow{2}{*}{10,10} & 0.4997 & 0.4997 & 0.4971 & 0.5024 & 0.5013 & 0.4985 & 0.5041 & 0.4992 & 0.4964 & 0.5020 \\
& 0.0116 & 0.0073 & 0.0073 & 0.0073 & 0.0107 & 0.0107 & 0.0107 & 0.0108 & 0.0108 & 0.0108 \\
\multirow{2}{*}{15,15} & 0.4998 & 0.4998 & 0.4980 & 0.5017 & 0.4990 & 0.4971 & 0.5009 & 0.5009 & 0.4990 & 0.5028 \\
& 0.0082 & 0.0059 & 0.0060 & 0.0060 & 0.0075 & 0.0076 & 0.0075 & 0.0076 & 0.0076 & 0.0076 \\
20,20 & 0.4998 & 0.4998 & 0.4984 & 0.5013 & 0.5001 & 0.4985 & 0.5016 & 0.5005 & 0.4992 & 0.5019 \\
& 0.0061 & 0.0048 & 0.0050 & 0.0049 & 0.0059 & 0.0060 & 0.0061 & 0.0058 & 0.0060 & 0.0060 \\
\hline
\end{tabular}


Table 3: Average estimates (first row) and MSE's (second row) of the stress-strength reliability $(R=0.6)$ for different variation of $n, m$ when $\alpha_{1}=2$ and $\alpha_{2}=2$

\begin{tabular}{ccccccccccc}
\hline \multirow{2}{*}{$\mathrm{n}, \mathrm{m}$} & MLE & \multicolumn{3}{c}{ Bayes1 } & \multicolumn{3}{c}{ Bayes2 } & \multicolumn{3}{c}{ Bayes0 } \\
\cline { 2 - 11 } & $\hat{R}_{M L}$ & $\hat{R}_{B S}$ & $\hat{R}_{B L}$ & $\hat{R}_{B L 1}$ & $\hat{R}_{B S}$ & $\hat{R}_{B L}$ & $\hat{R}_{B L 1}$ & $\hat{R}_{B S}$ & $\hat{R}_{B L}$ & $\hat{R}_{B L 1}$ \\
\hline \multirow{2}{*}{5,5} & 0.5930 & 0.5552 & 0.5505 & 0.5599 & 0.5825 & 0.5776 & 0.5873 & 0.5845 & 0.5795 & 0.5893 \\
& 0.0213 & 0.0093 & 0.0098 & 0.0089 & 0.0178 & 0.0181 & 0.0175 & 0.0187 & 0.0190 & 0.0184 \\
\multirow{2}{*}{10,10} & 0.5983 & 0.5732 & 0.5707 & 0.5758 & 0.5898 & 0.5871 & 0.5924 & 0.5913 & 0.5887 & 0.5939 \\
& 0.0109 & 0.0068 & 0.0069 & 0.0066 & 0.0101 & 0.0102 & 0.0101 & 0.0104 & 0.0105 & 0.0103 \\
\multirow{2}{*}{15,15} & 0.5971 & 0.5792 & 0.5773 & 0.5810 & 0.5915 & 0.5896 & 0.5934 & 0.5938 & 0.5921 & 0.5955 \\
& 0.0074 & 0.0054 & 0.0055 & 0.0053 & 0.0070 & 0.0071 & 0.0070 & 0.0072 & 0.0073 & 0.0072 \\
20,20 & 0.5971 & 0.5831 & 0.5819 & 0.5842 & 0.5952 & 0.5936 & 0.5966 & 0.5940 & 0.5925 & 0.5953 \\
& 0.0056 & 0.0045 & 0.0047 & 0.0048 & 0.0055 & 0.0058 & 0.0058 & 0.0056 & 0.0058 & 0.0059 \\
\hline
\end{tabular}

Table 4: The values of different statistical measures for the data set I and II respectively.

\begin{tabular}{cccccc}
\hline \multicolumn{5}{c}{ Data Set-I } \\
\hline MODEl & ESTIMATE & AIC & BIC & -LOGL & KS \\
\hline GIED & $(0.7770681,49.2410155)$ & 773.1815 & 777.3024 & 384.5908 & 0.245329 \\
IRD & 741.3652 & 840.1341 & 842.066 & 419.0671 & 0.6039 \\
IED & 59.12589 & 773.3742 & 775.4346 & 385.6871 & 0.287505 \\
\hline \multicolumn{7}{c}{ Data Set-II } & & & \\
\hline GIED & $(1.1799,83.8998)$ & 572.4309 & 576.0443 & 284.215 & 0.1901 \\
IRD & 2547.4170 & 962.7151 & 964.4993 & 480.3576 & 0.3783 \\
IED & 75.3793 & 571.0622 & 572.8689 & 284.5311 & 0.1823 \\
\hline
\end{tabular}

Table 5: Summary of the data set I and data set II

\begin{tabular}{ccccccc}
\hline \multicolumn{7}{c}{ Descriptive Statistics } \\
\hline Data & Min. & $Q_{1}$ & Median & Mean & $Q_{3}$ & Max. \\
\hline I & 6.53 & 83.25 & 151.5 & 226.2 & 237 & 1417 \\
II & 12.2 & 63.47 & 127 & 219.5 & 209 & 1776 \\
\hline
\end{tabular}

